Revista Universo Contábil, ISSN 1809-3337
Blumenau, v. 16, n. 3, p. 121-139, jul./set., 2020

\title{
MECANISMOS DE GOVERNANÇA CORPORATIVA E RECOMENDAÇÕES DOS ANALISTAS DE MERCADO: NOVAS EVIDÊNCIAS A PARTIR DA PERSPECTIVA DA TEORIA DA SINALIZAÇÃO
}

\author{
CORPORATE GOVERNANCE MECHANISMS AND MARKET ANALYSTS' \\ RECOMMENDATION: NEW EVIDENCES FROM THE SIGNALING THEORY
}

\section{MECANISMOS DE GOBIERNO CORPORATIVO Y RECOMENDACIONES DEL ANALISTA DE MERCADO: NUEVA EVIDENCIA DE LA PERSPECTIVA DE LA TEORÍA DE LA SEÑALIZACIÓN}

Recebido em: 07-11-2019

Avaliado em: 05-08-2020

Reformulado em: 18-05-2021

Aceito para publicação em: 04-06-2021

Publicado em: 21-06-2021

Editor Responsável: Moacir M. Rodrigues Jr.

\author{
Flávia Zóboli Dalmácio ${ }^{1}$ \\ Amaury José Rezende ${ }^{2}$ \\ Rogiene Batista dos Santos ${ }^{3}$
}

\section{RESUMO}

O objetivo deste artigo é analisar, a partir da Teoria da Sinalização, se estar nos níveis diferenciados de governança corporativa afeta as recomendações dos analistas de investimento do mercado brasileiro. Essa teoria trata dos problemas de assimetria informacional, que podem ser reduzidos com a sinalização de mais informação. Como proxies para a adoção de práticas diferenciadas de governança corporativa utilizou-se a listagem das empresas que adotaram níveis diferenciados de governança corporativa da Brasil, Bolsa, Balcão (B3). As informações sobre as recomendações de compra, de manutenção e de venda foram coletadas da base de dados Thomson Reuters. No total, foram analisadas 450 empresas brasileiras de capital aberto com ações negociadas na B3, no período de 2010 a 2016, totalizando 3.112 observações. Dessas, 185 empresas eram cobertas por analistas, totalizando 1.034 observações, e 265 empresas não eram cobertas por analistas, perfazendo 2.078 observações. Para análise dos dados, utilizou-se o método de Regressão Logística. De acordo com os resultados obtidos, há evidências de que os níveis diferenciados de governança corporativa afetam as recomendações de compra e venda dos analistas. Portanto, esta é uma evidência de que esses segmentos de listagem podem ser ententidos com um sinal positivo para os analistas. Desta forma, esta evidência sugere que a adoção de boas práticas de governança corporativa seja valorizada por analistas e investidores do mercado brasileiro. Os resultados encontrados podem auxiliar investidores, gestores, reguladores e acadêmicos na compreensão acerca de como a governança pode ser um instrumento de sinalização positiva no mercado financeiro.

Palavras-chave: Governança Corporativa. Recomendações. Analistas. Sinalização.

\footnotetext{
1 Doutora em Controladoria e Contabilidade pela FEA/USP; Professora do Departamento de Contabilidade da FEARP/USP; E-mail: flaviazd@usp.br

${ }^{2}$ Doutor em Controladoria e Contabilidade pela FEA/USP; Professor Associado do Departamento de Contabilidade da FEARP/USP; E-mail: amauryj@usp.br

${ }^{3}$ Doutora em Controladoria e Contabilidade pela FEARP/USP; Professora Associada no Pecege/MBA USP Esalq; Email: rogienebatista@alumni.usp.br
} 


\section{ABSTRACT}

This paper aims to analyze, based on the Signaling Theory, whether being at different levels of corporate governance affects the recommendations of investment analysts in the Brazilian market. This theory addresses the problems of informational asymmetry, which can be reduced by signaling more information. As proxies for adopting differentiated corporate governance practices, the list of companies that adopted differentiated levels of corporate governance from Brasil, Bolsa, Balcão (B3) was used. Information on purchase, maintenance, and sales recommendations was collected from the Thomson Reuters database. In total, 450 publicly traded Brazilian companies with shares traded on B3 from 2010 to 2016 were analyzed, totaling 3,112 observations, 185 companies covered by analysts, totaling 1,034 observations, and 265 companies not covered by analysts, totaling 2,078 observations. For data analysis, the Logistic Regression method was used. According to the data obtained, there is evidence that those at different levels of corporate governance affect analysts' buy and sell recommendations. Therefore, this is evidence that different levels of governance can be understood as a positive sign for analysts. Thus, this evidence suggests that the adoption of good corporate governance practices is valued by analysts and investors in the Brazilian market. The results can help investors, managers, regulators, and academics understand how governance can be a positive signaling instrument in the financial market.

Keywords: Corporate Governance. Recommendations. Analysts. Signaling.

\section{RESUMEN}

El propósito de este artículo es analizar, con base en la Teoría de la Señalización, si estar en diferentes niveles de gobierno corporativo afecta las recomendaciones de los analistas de inversión en el mercado brasileño. Esta teoría se ocupa de problemas de asimetría de la información, que se pueden reducir mediante la señalización de más información. Como proxy para la adopción de prácticas diferenciadas de gobierno corporativo, se utilizó la lista de empresas que adoptaron niveles diferenciados de gobierno corporativo de Brasil, Bolsa, Balcão (B3). La información sobre recomendaciones de compra, mantenimiento y ventas se obtuvo de la base de datos de Thomson Reuters. En total, se analizaron 450 empresas brasileñas que cotizan en bolsa con acciones negociadas en B3 de 2010 a 2016, totalizando 3.112 observaciones. 185 empresas fueron cubiertas por analistas, totalizando 1.034 observaciones y 265 empresas no cubiertas por analistas, totalizando 2.078 observaciones. Para el análisis de los datos se utilizó el método de Regresión Logística. Según los datos obtenidos, existe evidencia de que quienes se encuentran en diferentes niveles de gobierno corporativo afectan las recomendaciones de compra y venta de los analistas. Por tanto, esta es una evidencia de que los diferentes niveles de gobernanza pueden entenderse como una señal positiva para los analistas. Así, esta evidencia sugiere que la adopción de buenas prácticas de gobierno corporativo es valorada por analistas e inversionistas en el mercado brasileño. Los resultados encontrados pueden ayudar a los inversores, administradores, reguladores y académicos a comprender cómo la gobernanza puede ser un instrumento de señalización positiva en el mercado financiero.

Palabras-clave: Gobierno corporativo. Recomendaciones. Analistas. Señalización

\section{INTRODUÇÃO}

O desenvolvimento da tecnologia e da ciência ampliou a disseminação da informação (Yin \& Tan, 2017). Assim, a informação acerca das práticas de governança corporativa pode ser entendida pelo mercado como um sinal. As pesquisas empíricas têm apresentado alguns indícios de que ao adotar níveis diferenciados de governança corporativa, a empresa emite um sinal positivo ao mercado, de forma a influenciar a precisão das previsões dos analistas de investimento do mercado brasileiro. Além disso, alguns pesquisadores têm encontrado que esse sinal afeta a probabilidade condicional 
que definem as crenças, tanto para os analistas que elaboram as previsões, quanto para os investimentos no momento de diversificar seus investimentos (Dalmácio, 2009; Dalmácio, Lopes, \& Rezende, 2010; Dalmácio, Lopes, Rezende, \& Sarlo Neto, 2013).

Bhat, Hope e Kang (2006) investigaram como as diferenças na transparência em 21 países afetavam a acurácia dos analistas para as firmas desses países. Esses autores analisaram se a governança corporativa afetava a acurácia na previsão dos analistas. Encontraram que a governança corporativa era positivamente relacionada com a acurácia na previsão dos analistas. Além disso, os resultados sugerem que a governança corporativa tem maior significância quando o ambiente legal é fraco. Tendo em vista o contexto legal do Brasil, espera-se que os níveis de governança corporativa exerçam um papel significativo na previsão dos analistas.

Os analistas, nesse contexto, exercem uma função significativa, porque captam e interpretam esses sinais e auxiliam os investidores quanto às possibilidades de investimentos (Healy \& Palepu, 2001; Martinez, 2004a; Yu, 2005). Desse modo, os analistas de investimento atuam como intermediários informacionais, entre empresas e investidores, e são agentes propagadores de informações (Martinez, 2004a; 2004b), pois coletam e consolidam as informações (obrigatórias ou voluntárias) divulgadas pelas empresas, avaliam a performance dessas empresas, preveem os resultados futuros e recomendam o que os investidores devem fazer: comprar, vender ou manter as ações (Healy \& Palepu, 2001; Boff, Procianoy, \& Hoppen, 2006; Martinez, 2004a; 2007).

Contudo, há evidências na literatura de que as recomendações dos analistas não produzem retornos anormais e nem alcançam a performance de benchmarks passivos, em termos médios (Cowles, 1933; Jaffe \& Mahoney, 1999; Desai, Liang, \& Singh, 2000). Martinez (2004a, p. 60) ressalta que "isso não significa que um analista não possua a capacidade de escolher uma boa ação", entretanto, "identificar um analista de desempenho superior" cujas recomendações possuam valor para os investidores é o grande desafio.

Neste cenário, a governança corporativa é entendida com um sinal que pode influenciar a precisão das previsões dos analistas (Dalmácio, 2009; Dalmácio et al., 2010; Dalmácio et al., 2013). Pode, também, ser capaz de influenciar as recomendações emitidas por esses analistas. Segundo Larcker, Richardson e Tuna (2007), há escassez de uma teoria bem elaborada com relação a natureza multidimensional da governança corporativa. Essa ausência dificulta a especificação de modelos estruturais apropriados, além da determinação de quais construtos relevantes de governança corporativa poderiam ser incluídos nas análises. Tendo em vista a escassez de uma base teórica para a governança corporativa, buscou-se a aplicação dos pressupostos da Teoria da Sinalização (Spence, 1973), de forma a ampliar e aprofundar a compreensão acerca da relação entre a recomendação dos analistas e as boas práticas de governança corporativa.

A utilização da Teoria da Sinalização é justificada porque, uma vez, que as empresas emitem sinais ao adotarem níveis diferenciados de governança corporativa, eles podem ser considerados como parâmetros na alteração da probabilidade condicional que determinam as crenças, tantos dos analistas quanto dos investidores do mercado brasileiro (Dalmácio, 2009; Dalmácio et al., 2010; Dalmácio et al., 2013).

No Brasil, a então Bolsa de Valores de São introduziu em dezembro de 2000 os níveis diferenciados de governança corporativa, com o objetivo de permitir a comparação entre as empresas listadas no país. Esses níveis de governança exigem das empresas maior transparência, que varia de nível para nível. Os três níveis de governança corporativa são: Níveis Diferenciados de Governança Corporativa (Nível 1 e Nível 2) e o Novo Mercado. Alguns estudos têm analisado estes níveis. Por exemplo, Silva, Silveira, Corso e Stadler (2011) investigaram se havia diferenças entre as empresas que aderiram às práticas de governança corporativa antes e após a efetiva adesão aos níveis diferenciados. Oliveira (2019) verificou os possíveis impactos da governança nos índices econômicos das empresas. Por outro lado, Marcos, Nascimento, Nez e Kroenke (2018) analisaram a relação entre o grau de internacionalização e o desempenho econômico-financeiro das empresas listadas no Novo 
Mercado da B3. Mais recentemente, Marquiori (2019) analisou se a migração de nível de listagem de governança corporativa na Bolsa de Valores reflete numa apreciação das ações.

Assim, o presente estudo contribui para a literatura ao analisar se os níveis de governança da B3 afetam a recomendação dos analistas, a partir da Teoria da Sinalização. Dessa forma, esta pesquisa busca responder à seguinte questão: As empresas, ao adotarem níveis diferenciados de governança corporativa da B3, influenciam as recomendações dos analistas de investimentos? Assim, o objetivo geral desta pesquisa é verificar, a partir da Teoria da Sinalização, a relação entre governança e recomendação dos analistas no mercado brasileiro.

Outro fator de relevância para esta pesquisa é que a atividade exercida pelos analistas de mercado pode auxiliar na redução dos custos de agência, relacionados à separação da propriedade e do controle (Jensen \& Meckling, 1976; Moyer, Chatfield, \& Sisneros, 1989; Chung \& Jo, 1996). Além disso, os analistas de mercado têm uma função significativa, se eles considerarem os níveis diferenciados de governança em suas recomendações de compra e venda das ações (Silveira, 2004, p. 211).

A literatura apresenta alguns estudos que analisaram a relação entre transparência financeira e acurácia na previsão dos analistas. Porém, poucos foram desenvolvidos de forma a evidenciar se a governança corporativa afeta a recomendação dos analistas. Portanto, não há consenso na literatura se a governança corporativa, de fato, influencia a recomendação dos analistas. Desta forma, a principal contribuição deste artigo deve-se à utilização da Teoria da Sinalização como um arcabouço teórico que pode lançar luz acerca da relação entre governança corporativa e recomendação dos analistas.

Os resultados encontrados mostram que, de forma geral, estar classificado em algum nível de governança corporativa afeta a recomendação dos analistas. Confirma-se, assim, a hipótese de que a governança corporativa é um sinal positivo. Portanto, os resultados encontrados podem auxiliar investidores, gestores, reguladores e acadêmicos ao evidenciar que adotar práticas diferenciadas de governança corporativa pode ser um sinal positivo que a empresa emite ao mercado, inclusive para os analistas. Esse sinal mostra que a empresa está preocupada e investe em governança corporativa, o que pode reduzir a assimetria de informação entre a empresa e o mercado e, consequentemente, afetar a recomendação dos analistas.

\section{REFERENCIAL TEÓRICO}

\subsection{Teoria da Sinalização e Governança Corporativa}

Incialmente, a Teoria da Sinalização foi concebida para esclarecer problemas de informação no mercado de trabalho (Spence, 1973). Mas, ela também pode ser aplicada em qualquer mercado em que exista assimetria de informação (Morris, 1987). Dessa forma, a partir da Teoria da Sinalização, procurou-se verificar se os níveis de governança corporativa eram uma forma das empresas emitirem mais informação ao mercado e, portanto, uma maneira de emitirem um sinal positivo acerca de suas práticas de governança corporativa.

Spence (1973) apresentou um modelo conceitual a partir do qual características observáveis, tais como: grau de instrução, experiência de mercado, raça, sexo e demais características podem ser sinais que diferenciam os indivíduos no mercado de trabalho. Ele também constatou que a sinalização ocorre se os custos da mesma forem diferentes entre aqueles que enviam os sinais.

Sob a perspectiva dessa teoria, busca-se, neste artigo, verificar se as empresas sinalizam, por meio de suas práticas diferenciadas de governança corporativa, qualidade e credibilidade informacional, o que pode representar boas oportunidades de investimento. Ao adotarem práticas diferenciadas de governança corporativa, as empresas se diferenciam umas das outras no mercado de capitais, e possibilitam a realização dos investimentos (adaptado do exemplo de mercado de carros usados de Akerlof, 1970). 
De acordo com Shleifer e Vishny (1997, p. 738), "os mecanismos de governança corporativa são as instituições econômicas e legais que podem ser alteradas, por meio do processo político, às vezes, para melhor". Essa afirmação está alinhada com a suposição de que a governança pode representar um sinal no mercado de capitais. Ou seja, as empresas, mediante a incorrência de custos, investem em boas práticas de governança com o objetivo de comunicar algo ao mercado, principalmente, a analistas e investidores. Entretanto, essas empresas decidem instituir práticas de governança superiores quando seus benefícios são maiores que seus custos (Lopes, 2008).

A partir da Teoria da Sinalização, a adoção de níveis diferenciados de governança corporativa representa um sinal que a empresa emite ao mercado e que pode afetar a recomendação dos analistas e/ou dos investidores (Dalmácio, 2009; Dalmácio et al., 2010; Dalmácio et al., 2013). Assim, investigou-se se a recomendação dos analistas é afetada pela governança corporativa. O pressuposto subjacente é que boas estruturas de governança corporativa tendem a aumentar a transparência da empresa, melhorando, assim, a informação disponível aos analistas, o que conduz a melhor acurácia em suas previsões. Ou seja, muitas ações tomadas pelas empresas são motivadas, principalmente, pelo desejo de enviar um sinal positivo a outros agentes, pois, esse sinal pode reduzir a assimetria de informação entre os agentes.

Chiang \& Chia (2005) verificaram se a melhor transparência corporativa conduzia a melhor acurácia na previsão dos analistas. Os resultados indicaram que a acurácia na previsão dos analistas tinha relação significativa com a transparência corporativa. Além disso, seus resultados mostram que quanto maior era a transparência corporativa, maior era a acurácia na previsão dos analistas.

Nesse mesmo sentido, Byard, Li e Weintrop (2006) analisaram a relação entre governança corporativa e a qualidade de informação para os analistas. Eles constataram que havia uma relação linear entre a informação dos analistas sobre os lucros e a qualidade da governança. Os resultados também mostraram que a melhor governança corporativa era essencial para os usuários das informações financeiras, principalmente para os analistas.

Ahmad-Zaluki e Wan-Hussin (2010) examinaram os efeitos dos mecanismos de governança corporativa na acurácia da previsão dos analistas. Os autores investigaram se a governança corporativa era um sinal verossímil de melhoria da qualidade da informação financeira. Eles encontraram que empresas com maiores mecanismos de governança corporativa apresentavam maior acurácia na previsão dos analistas. Portanto, os resultados encontrados mostraram que uma efetiva governança corporativa era um sinal de melhor informação financeira

Dessa forma, quando a empresa decide estar em um dos níveis de governança, pressupõe-se que ela busca enviar um sinal positivo ao mercado, inclusive para os analistas. O presente estudo lança luz sobre a relação entre a sinalização e governança corporativa e seu efeito na recomendação dos analistas. Portanto, os resultados deste artigo contribuem para melhor compreensão de como que a governança é entendida como um sinal de melhor informação financeira por parte dos analistas.

\subsection{Recomendação dos Analistas}

Os analistas do mercado de capitais são profissionais que possuem habilidades e conhecimentos específicos, que os qualificam para executar com eficiência a escolha e o gerenciamento de investimentos (Franco, 2002; Yu, 2005). Esses profissionais, atentos ao que acontece no mercado, reúnem e examinam informações que as empresas divulgam, as consolidam, fazem avaliação acerca da performance das empresas, realizam previsões sobre resultados esperados e, então, fazem as suas recomendações (Healy \& Palepu, 2001; Martinez, 2004a; 2007).

As recomendações ao investidor podem ser classificadas em cinco grupos: (1) recomendações de compra forte; e (2) compra, quando o modelo de avaliação de uma empresa apura um valor maior que o preço de mercado; (3) recomendações de manutenção, quando o modelo de avaliação de uma empresa apura um valor equivalente ao preço de mercado; (4) recomendações de venda; e (5) venda forte, quando o modelo de avaliação de uma empresa apura um valor maior que o preço de mercado. 
As recomendações expressam a opinião dos analistas, no que se refere às expectativas de obtenção de retornos anormais pelas empresas analisadas, proporcionando ao investidor a oportunidade de realizar retornos futuros, mas também evitar perdas pela manutenção de um ativo depreciado. No entanto, essas recomendações podem se tornar obsoletas de um dia para outro, pois dependem do comportamento das ações no mercado (Martinez, 2004a; 2007).

A expectativa dos investidores é que as recomendações dos analistas de mercado agreguem valor aos seus investimentos, produzindo retornos anormais. Porém, essa é uma questão controversa na literatura internacional, pois existem pesquisas que evidenciaram que as recomendações não produzem retornos anormais (Cowles, 1933; Desai \& Jain, 1995; Jaffe \& Mahoney, 1999) e existem aquelas que evidenciaram o contrário, que em virtude de recomendações, foi obtido um excesso de retorno (Womack, 1996; Desai et al., 2000; Barber, Lehavy, \& Trueman, 2001). Portanto, não há consenso na literatura se as recomendações dos analistas afetam ou não o valor dos investimentos.

Segundo Martinez (2004a, p. 61), "o grande desafio dos investidores é justamente identificar quais são esses analistas cujas recomendações possuem valor efetivo". Além disso, deve-se considerar, na análise dos retornos anormais, os custos de transação, a fim de se obter um retorno anormal líquido (Barber et al., 2001). Vale ressaltar que as recomendações de consenso dos analistas de mercado podem deixar de apresentar as notícias ruins ou recomendações de vendas, ou seja, os analistas são relutantes em emitir recomendações de venda (Barber et al., 2001). Todavia, quanto maior o número de analistas que representam as expectativas do mercado, melhor é o indicador de desempenho futuro (Martinez, 2004a; 2007).

No mercado americano, Barber, Lehavy e Trueman (2000) identificaram que as recomendações de compras das maiores corretoras resultavam em um retorno anormal maior que as recomendações das corretoras menores e, em relação às recomendações de venda, era exatamente o contrário: as corretoras menores tinham um desempenho melhor. Os autores justificam que este segundo resultado é surpreendente e sugere que as recomendações de venda das grandes corretoras são menos oportunas.

Alguns pesquisadores, tais como, Jegadeesh e Kim (2006), Barber et al. (2001) e Cliff e Denis (2004) verificaram se a quantidade de recomendações de venda e de forte venda era muito menor que as recomendações de compra ou de forte compra nos países analisados. Além disso, a literatura mostra que a hesitação dos analistas de emitirem venda ou forte venda é em função do conflito de interesse, pois eles buscam ter uma boa reputação nas empresas.

Desai e Jan (1995), Womack (1996) e Barber, Lehavy, McNichols e Trueman (2006) verificaram se as recomendações de compra (buy) predominavam sobre as recomendações de venda (sell). No entanto, as grandes corretoras, geralmente, possuem um percentual menor dessas recomendações de compra. Emitir recomendações de venda representa mais risco aos analistas, porque elas são mais visíveis e menos frequentes. Um julgamento incorreto em uma recomendação de venda pode afetar a reputação de um analista mais do que uma recomendação incorreta de compra, porém, se os custos de emitir uma recomendação de venda forem maiores, então, a compensação do analista deve ser maior também (Womack, 1996, p. 165).

Groysberg, Healy, Serafeim e Shanthikumar (2013) analisaram a seleção e desempenho dos analistas buy-side comparando com analistas da outra ponta, sell-side. Os autores constataram que as recomendações possuíam uma volatilidade média de quase metade daquela observada nas recomendações dos analistas sell-side, e que as capitalizações de mercado tinham uma variação quase sete vezes maior.

Esses resultados podem sinalizar que os gestores de fundos (clientes de analistas buy-side) têm preferência de que os seus analistas cubram ações com menor volatilidade e que tenham maior liquidez. Além disso, os autores destacam que os analistas buy-side fazem recomendações mais pessimistas do que a sua contraparte sell-side. Este resultado está alinhado com a premissa de que analistas buy-side sofrem menos conflitos de interesse. 
No mercado brasileiro, Martinez (2004a; 2007), ao analisar dados do período de 1995 a 2003 , verificou que as recomendações de compra representaram, em média, mais de $50 \%$ das recomendações, com tendência de crescimento, tanto na perspectiva de recomendações individuais, quanto na de consenso. No que se refere às recomendações de venda, observou-se uma queda (12\%, em termos médios). Além disso, verificou-se que, "em termos médios, o desempenho das recomendações dos analistas é muito variável" (respectivamente, p. 177; p. 10) e que "o mercado brasileiro é muito mais sensível a revisões de recomendações para baixo, do que o é para as recomendações para cima" (respectivamente, p. 190; p. 13). Martinez (2004a) também investigou, no mercado brasileiro, o efeito nas recomendações emitidas: (1) do tamanho das corretoras; e (2) do tamanho das empresas analisadas. As evidências do estudo estão apresentadas na Tabela 1.

Tabela 1

Características que podem influenciar as recomendações dos analistas

\begin{tabular}{|c|c|c|c|}
\hline Efeito & Proxy & Hipótese & $\begin{array}{l}\text { Evidências } \\
\end{array}$ \\
\hline $\begin{array}{c}\text { Tamanho da } \\
\text { Corretora }\end{array}$ & $\begin{array}{l}\text { Número de } \\
\text { recomendações } \\
\text { emitidas pela } \\
\text { corretora no ano }\end{array}$ & $\begin{array}{l}\text { Quanto maior o } \\
\text { tamanho da corretora, } \\
\text { mais informativas as } \\
\text { recomendações (maior } \\
\text { o efeito nos preços). }\end{array}$ & $\begin{array}{l}\text { Os resultados evidenciam que o tamanho deve ser } \\
\text { levado em consideração, no entanto, nem tanto por } \\
\text { uma capacidade de previsão superior, "mas, } \\
\text { principalmente, por um aspecto de força } \\
\text { mercadológica que as grandes corretoras dispõem" } \\
\text { (p. 187). }\end{array}$ \\
\hline $\begin{array}{l}\text { Tamanho das } \\
\text { Empresas } \\
\text { Analisadas }\end{array}$ & $\begin{array}{l}\text { Valor de mercado } \\
\text { da empresa numa } \\
\text { unidade } \\
\text { monetária } \\
\text { constante. }\end{array}$ & $\begin{array}{l}\text { Quanto menor o } \\
\text { tamanho das empresas } \\
\text { analisadas, maior o } \\
\text { efeito nos preços das } \\
\text { recomendações de } \\
\text { compra e venda. }\end{array}$ & $\begin{array}{l}\text { No caso das recomendações de venda, as pequenas } \\
\text { empresas possuem um desempenho superior às } \\
\text { grandes empresas. } \\
\text { "No que se refere às recomendações de compra, no } \\
\text { momento inicial, os retornos das pequenas } \\
\text { empresas são maiores, mas, à medida que se avança } \\
\text { no tempo, essas recomendações parecem perder } \\
\text { fôlego, resultando, num prazo de } 120 \text { dias, num } \\
\text { rendimento inferior ao das recomendações de } \\
\text { compra para grandes empresas" (p. 187). }\end{array}$ \\
\hline Rentabilidade & $\begin{array}{l}\text { ROA. Retorno } \\
\text { sobre o Ativo. }\end{array}$ & $\begin{array}{l}\text { Quanto mais rentáveis } \\
\text { as empresas forem, } \\
\text { maior será a chance de } \\
\text { ela ser recomendada }\end{array}$ & $\begin{array}{l}\text { "O analista deve conhecer a natureza do produto, } \\
\text { antecipando a demanda dos clientes; compreender } \\
\text { a importância do fator humano no negócio; possuir } \\
\text { a sensibilidade para identificar a margem de } \\
\text { rentabilidade do negócio." (p. 17) }\end{array}$ \\
\hline Liquidez & Liquidez geral. & $\begin{array}{l}\text { Empresas que são } \\
\text { recomendadas pelos } \\
\text { analistas são mais } \\
\text { líquidas. }\end{array}$ & $\begin{array}{l}\text { "Está amplamente evidenciado na literatura que o } \\
\text { aumento da cobertura pelos analistas em relação a } \\
\text { uma determinada ação promove uma melhoria na } \\
\text { liquidez e um efeito positivo no preço." (p. 62) }\end{array}$ \\
\hline
\end{tabular}

Fonte: Adaptado de Martinez (2004a).

\subsection{Governança Corporativa e Recomendação dos Analistas}

As principais pesquisas que se propuseram em analisar a relação entre governança corporativa e recomendação dos analistas de mercado foram desenvolvidas por Yu (2011), Lin e Tai (2013) e Farooq e Amrani (2013). Yu (2011), ao examinar se as recomendações dos analistas eram influenciadas pela força da governança corporativa nos mercados emergentes para uma amostra de 805 empresas em 26 países de mercados emergentes, verificou que os analistas tendiam a emitir recomendações favoráveis para empresas com melhores mecanismos de governança corporativa. Além disso, esta evidência está concentrada em países onde a proteção do investidor é relativamente fraca.

Lin e Tai (2013) examinaram, a partir de 55.652 recomendações de empresas listadas na Bolsa de Valores de Taiwan, como os analistas recomendariam empresas com governança ruim em um mercado emergente, em que a assimetria de informação tende a ser alta e os direitos dos acionistas 
não estão bem protegidos por sistemas legais. De acordo com os resultados obtidos, as recomendações de compra de analistas em empresas com menor governança corporativa são menos confiáveis. Além disso, melhorar a governança corporativa não só pode reduzir os problemas das agências dentro das empresas, mas também melhorar a qualidade da informação produzida pelos analistas e reduzir o risco de informação dos investidores.

Assim, de acordo com as premissas da Teoria da Sinalização e na questão de pesquisa deste artigo, a hipótese deste trabalho é que H1: Empresas que adotam práticas diferenciadas de governança corporativa afetam a recomendação dos analistas. Alguns artigos também verificaram o efeito da governança corporativa na dispersão dos analistas. Por exemplo, Farooq e Amrani (2013) documentaram o efeito dos mecanismos de governança corporativa sobre a dispersão nas recomendações dos analistas nos mercados emergentes asiáticos. Os autores verificaram uma dispersão significativamente maior nas recomendações dos analistas durante o período pré-crise (regime de governança pobre) em relação ao período pós-crise (melhor regime de governança).

Segundo os autores, os resultados mostram que os mecanismos de governança corporativa podem explicar a maior parte da diferença de dispersão entre os dois períodos. Alguns estudos anteriores verificaram a relação entre transparência financeira e a acurácia na previsão dos analistas. Mas, a análise entre a governança corporativa e a acurácia na previsão dos analistas ainda é uma questão em aberto.

\section{METODOLOGIA}

A fim de atingir o objetivo deste artigo, utilizou-se uma abordagem metodológica com características de pesquisa positiva, baseada em conceitos da Teoria da Sinalização (Spence, 1973). O positivismo clássico busca a "explicação de fenômenos a partir da identificação de suas relações", além de considerar "imprescindível a existência de uma teoria para nortear as observações" (Martins \& Theóphilo, 2007, p. 41). Assim, buscou-se, empiricamente, analisar a relação entre adoção de níveis diferenciados de governança corporativa e as recomendações dos analistas de investimento do mercado brasileiro.

Os dados foram empilhados em painel, de forma ser possível a verificação do efeito da governança corporativa nas recomendações dos analistas ao longo do tempo. Essa escolha metodológica é justificada porque a amostra deste estudo é composta por um conjunto de dados com dimensão transversal e temporal, dado que a amostra de empresas $(i=1,2, \ldots, 450)$ será observada ao longo do tempo $(\mathrm{t}=2010, \ldots, 2016)$. De acordo com Wooldridge (2010), dessa forma, os dados dispostos em corte transversal e em série temporal são referenciados na literatura econométrica como dados em painel ou dados longitudinais. De forma a atingir o objetivo desta pesquisa, utilizou-se uma amostra de dados em painel com informações das empresas brasileiras financeiras e não-financeiras de capital aberto listadas na B3 no período de 2010 a 2016. Conforme a Tabela 2, observa-se que 450 empresas compõem a amostra de trabalho, totalizando 3.112 observações.

Tabela 2

Amostra de trabalho

\begin{tabular}{lccc}
\hline & Amostra Inicial & Ausência de Dados & Amostra de Trabalho \\
\hline Empresas & 588 & 138 & 450 \\
Observações & 3.579 & 467 & 3.112 \\
\hline
\end{tabular}

Na Tabela 3 apresenta-se a lista dos setores de atuação das empresas que compõem a amostra, conforme classificação feita no banco de dados Thomson Reuters ${ }^{\circledR}$. A amostra total é composta com 3.112 observações de 450 empresas, sendo que 185 empresas têm cobertura de analistas, totalizando 1.034 observações. Por outro lado, 265 empresas não têm cobertura de analistas, perfazendo 2.078 
observações. De acordo com a Tabela 3, tanto no conjunto de empresas que têm cobertura, quanto naquelas que não tem cobertura de analistas, o setor em que há mais observações é o setor finanças.

Tabela 3

Lista dos setores de atuação das empresas da amostra de trabalho

\begin{tabular}{|c|c|c|c|c|c|}
\hline \multirow[b]{2}{*}{ TRBC Economic Sector Name (Thomson) } & \multicolumn{2}{|c|}{$\begin{array}{c}\text { Com cobertura de } \\
\text { analistas }\end{array}$} & \multicolumn{2}{|c|}{$\begin{array}{c}\text { Sem cobertura de } \\
\text { analistas }\end{array}$} & \multirow{2}{*}{$\begin{array}{c}\text { Amostra } \\
\text { total }\end{array}$} \\
\hline & Obs. & $\%$ & Obs. & $\%$ & \\
\hline Materiais básicos & 87 & $8 \%$ & 193 & $9 \%$ & 280 \\
\hline Consumo cíclico & 177 & $17 \%$ & 163 & $8 \%$ & 340 \\
\hline Varejistas de produtos cíclicos de consumo & 21 & $2 \%$ & 7 & $0 \%$ & 28 \\
\hline Consumo não cíclico & 87 & $8 \%$ & 109 & $5 \%$ & 196 \\
\hline Energia & 0 & $0 \%$ & 265 & $13 \%$ & 265 \\
\hline Finanças & 303 & $29 \%$ & 649 & $31 \%$ & 952 \\
\hline Cuidados de saúde & 8 & $1 \%$ & 6 & $0 \%$ & 14 \\
\hline Industriais & 136 & $13 \%$ & 93 & $4 \%$ & 229 \\
\hline Tecnologia & 14 & $1 \%$ & 0 & $0 \%$ & 14 \\
\hline Serviços de telecomunicações & 14 & $1 \%$ & 228 & $11 \%$ & 242 \\
\hline Serviços de utilidade pública & 187 & $18 \%$ & 365 & $18 \%$ & 552 \\
\hline TOTAL & 1.034 & $100 \%$ & 2.078 & $100 \%$ & 3.112 \\
\hline
\end{tabular}

A Tabela 4 apresenta a amostra dividida entre empresas com governança, que adotam algum dos três níveis diferenciados de governança da B3, e as empresas que não têm governança, ou seja, aquelas que não adotaram nenhum dos níveis de governança da B3. Das 450 empresas da amostra, 132 adotam algum dos níveis de governança, perfazendo 915 observações; sendo que 318 empresas não adotaram, totalizando 2.197 empresas.

Tabela 4

Amostra com governança e sem governança

\begin{tabular}{|c|c|c|c|c|c|}
\hline \multirow[b]{2}{*}{ TRBC Economic Sector Name (Thomson) } & \multicolumn{2}{|c|}{ Com governança } & \multicolumn{2}{|c|}{ Sem governança } & \multirow{2}{*}{ Amostra total } \\
\hline & Obs. & $\%$ & Obs. & $\%$ & \\
\hline Materiais básicos & 83 & $9 \%$ & 197 & $9 \%$ & 280 \\
\hline Consumo cíclico & 127 & $14 \%$ & 213 & $10 \%$ & 340 \\
\hline Varejistas de produtos cíclicos de consumo & 7 & $1 \%$ & 21 & $1 \%$ & 28 \\
\hline Consumo não cíclico & 111 & $12 \%$ & 85 & $4 \%$ & 196 \\
\hline Energia & 0 & $0 \%$ & 265 & $12 \%$ & 265 \\
\hline Finanças & 272 & $30 \%$ & 680 & $31 \%$ & 952 \\
\hline Cuidados de saúde & 14 & $2 \%$ & 0 & $0 \%$ & 14 \\
\hline Industriais & 105 & $11 \%$ & 124 & $6 \%$ & 229 \\
\hline Tecnologia & 7 & $1 \%$ & 7 & $0 \%$ & 14 \\
\hline Serviços de telecomunicações & 14 & $2 \%$ & 228 & $10 \%$ & 242 \\
\hline Serviços de utilidade pública & 175 & $19 \%$ & 377 & $17 \%$ & 552 \\
\hline TOTAL & 915 & $100 \%$ & 2.197 & $100 \%$ & 3.112 \\
\hline
\end{tabular}

Neste estudo, foram utilizadas três variáveis dependentes. A primeira é a variável dummy DREC_C $\mathrm{C}_{\mathrm{i}}$, que assume o valor igual a 1 se as ações da empresa $i$ receberam recomendações de compra no ano $t$; e igual a 0 , caso contrário. A segunda variável dependente é a variável dummy DREC_ $M_{\mathrm{it}}$, que assume o valor igual a 1 se as ações da empresa $i$ receberam recomendações de manutenção no ano $t$; e igual a 0 , caso contrário. Por fim, a terceira variável dependente é a dummy DREC_V $\mathrm{V}_{\mathrm{it}}$, igual a 1 se as ações da empresa $i$ receberam recomendações de venda no ano $t$; e igual a 0 , caso contrário.

Com relação às práticas diferenciadas de Governança Corporativa da B3, adotadas pelas empresas da amostra, seguiu-se a seguinte classificação: Nível 1, Nível 2 ou Novo Mercado. Estas são as variáveis independentes. Esses níveis são segmentos especiais de listagem e foram criados em 
dezembro de 2000, com o intuito de oferecer aos investidores melhores práticas de governança corporativa. Para que a empresa esteja em um desses níveis, é necessário que ela cumpra determinadas exigências (Brasil, Bolsa, Balcão, 2019).

Este conjunto de práticas diferenciadas pode ser representado por três variáveis binárias (dummies), que assumem valor igual a 1 (um) quando a empresa participa de algum nível diferenciado de prática de Governança Corporativa da Bovespa e 0 (zero) quando não participa: DBOV_N1 representa a prática de Governança Corporativa classificada como Nível 1; DBOV_N2 representa a prática de Governança Corporativa classificada como Nível 2; DBOV_NM representa a prática de Governança Corporativa classificada como Novo Mercado. A variável Recom*Govern é uma variável de interação entre ser recomendada pelos analistas e ter aderido níveis diferenciados de governança. Se a empresa recebeu alguma recomendação nos anos analisados, a variável Recom será igual a 1 (um); caso contrário, ela será 0 (zero). Se a empresa fez adoção de níveis diferenciados, a variável Govern será igual a 1 (um); caso contrário, ela será 0 (zero).

As variáveis de controle foram consideradas nos modelos, com o objetivo de separar seus efeitos sobre a variável dependente. Tomou-se por base a literatura sobre os determinantes das recomendações dos analistas de mercado, apresentam-se as variáveis de controle que foram utilizadas nos modelos.

1. Cobertura dos Analistas (QANALIST): consiste na quantidade de analistas que acompanham a empresa $i$ no período $t$. Há evidências de que quanto maior o número de analistas que representam as expectativas do mercado, melhor é o indicador de desempenho futuro (Martinez, 2004a, 180; 2007, p. 11).

2. Tamanho (TAMEMP): utilizou-se o logaritmo natural do valor de mercado da empresa $i$ no período $t$ como proxy para tamanho. Há evidências de que quanto menor o tamanho das empresas analisadas, maior o efeito nos preços das recomendações de compra e venda (Martinez, 2004a).

$$
T A M E M P_{i . t}=\ln \left(V M_{i, t}\right)
$$

3. Setor de Atividade (DSETOR): Representado por um conjunto de variáveis binárias (dummies), que assumem valor igual a 1 (um) para as empresas que participam de determinado setor e 0 (zero) para as empresas de outros setores.

4. Tamanho da Corretora (TAMCOR): representado pelo número de recomendações lançadas em um determinado período $t$ pela corretora $j$. Há evidências de que quanto maior o tamanho da corretora, mais informativas as recomendações (Martinez, 2004a).

As variáveis utilizadas neste estudo foram extraídas tanto da literatura nacional quanto internacional (Chung \& Jo, 1996; Bhat et al., 2006; Silva et al., 2011; Dalmácio et al., 2013; Oliveira, 2019; Marcos et al., 2018; Marquiori, 2019). Com o objetivo de tratar os outliers, as variáveis contínuas foram winsorizadas ao nível de $2,5 \%$ e $97,5 \%$.

Os dados e as informações utilizados nesta pesquisa foram coletados a partir das fontes apresentadas na Tabela 5. O primeiro passo para a análise dos dados foi analisar a matriz de correlação de Pearson. Constatou-se que todos os níveis de governança têm correlação positiva e significativa com a recomendação de compra da seguinte maneira: nível 1 correlação de 0,1626 , nível 2 correlação de 0,1182 e Novo Mercado correlação de 0,3044. Assim, esta é uma primeira evidência de que a governança afeta a recomendação de compra dos analistas. Com relação à recomendação de manutenção, verificou-se que para todos os níveis de governança houve correlação positiva e significativa com a recomendação de manutenção. Os resultados foram: nível 1 correlação de 0,1328, nível 2 correlação de 0,0662 e Novo Mercado correlação de 0,2644. Com relação à recomendação de venda, não se verificou um consenso nos resultados. Constatou-se que para o nível 1 houve correlação positiva e significativa de 0,0793 e para o Novo Mercado também uma correlação positiva e significativa de 0,1067. Porém, constatou-se relação negativa e significativa para o nível 2 (-0,0123). 
Tabela 5

Fontes dos dados utilizados nesta pesquisa

\begin{tabular}{|c|c|}
\hline Dados & Fontes \\
\hline $\begin{array}{c}\text { Recomendações dos analistas de empresas brasileiras de } \\
\text { capital aberto }\end{array}$ & $\begin{array}{c}\text { Banco de dados do sistema Thomson Reuters One - } \\
\text { Advanced Analytics }{ }^{\circledR}\end{array}$ \\
\hline $\begin{array}{c}\text { Econômico-Financeiros e de Mercado das empresas } \\
\text { brasileiras de capital aberto }\end{array}$ & $\begin{array}{c}\text { Banco de dados do sistema Thomson Reuters One - } \\
\text { Advanced Analytics }{ }^{\circledR}\end{array}$ \\
\hline $\begin{array}{c}\text { Práticas diferenciadas de governança corporativa das } \\
\text { empresas brasileiras de capital aberto (Níveis de } \\
\text { Governança da Bovespa) }\end{array}$ & Banco de dados da Brasi, Bolsa, Balcão (B3) \\
\hline
\end{tabular}

O objetivo central da presente pesquisa é analisar, a partir da Teoria da Sinalização, se estar nos níveis diferenciados de governança corporativa afeta as recomendações dos analistas de mercado. Para atingir o objetivo principal e testar as hipóteses elaboradas, foram utilizados modelos de regressão logística. No modelo logístico, os valores de uma série de variáveis independentes são usados para predizer a ocorrência de um evento (variável dependente).

A decisão pela análise de regressão logística (LOGIT), associada à Razão de Chances (OddsRatio), se justifica pela necessidade de estimar a probabilidade de empresas que adotaram níveis diferenciados de governança da B3, considerando um conjunto de variáveis explanatórias com base na literatura nacional e internacional.

De acordo com Hair, Anderson, Tatham e Black (1998), em função da natureza categórica binária da variável dependente, recomenda-se o uso da LOGIT pelo benefício de apresentar os resultados em probabilidades, classificar as observações em grupos, ter interpretação similar à regressão linear, exigir poucos pressupostos estatísticos para os dados, como o comportamento probabilístico de normalidade multivariada, e conter alta confiabilidade.

Primeiro, esta técnica calcula a razão entre a probabilidade de ocorrência e não-ocorrência de um evento constante na amostra, denominada razão de chances (Odds-Ratio). Em seguida, realiza a transformação logarítmica dessa razão. A estimação dos coeficientes é realizada pelo critério da máxima verossimilhança, de forma a obter a melhor regra de classificação associada ao evento.

O modelo da função logística calcula a probabilidade do efeito pela seguinte fórmula:

$$
P(X=1)=\frac{1}{1+e^{-\left(a+\sum \beta_{i} X_{i}+\sum \beta_{n} X_{n}\right)}}
$$

Os termos $a$ e $\beta_{i}$ são os parâmetros desconhecidos que foram estimados com base nos dados amostrais, obtidos a partir do método da máxima verossimilhança. Dessa forma, ao conhecer os parâmetros $a$ e $\beta_{i}$ e os valores das variáveis independentes para cada empresa, aplica-se a fórmula acima para calcular a probabilidade de que esta empresa receba uma recomendação $-P(X=1)$. $\mathrm{O}$ relacionamento entre a adoção de práticas diferenciadas de governança corporativa e as recomendações dos analistas de mercado pode ser expresso por:

RECOMENDAÇÃO = $f$ (práticas diferenciadas de governança corporativa, variáveis de controle)

Os três modelos (compra, manutenção e venda) que foram utilizados para verificar e analisar esse relacionamento, são apresentados, a seguir. O primeiro modelo testado foi o Modelo 1 Recomendação de compra. O objetivo deste modelo é testar a hipótese $\mathrm{H}_{1}$. Ou seja, verificar se as empresas que adotam práticas diferenciadas de governança corporativa têm mais probabilidade de receber recomendações de compra.

$$
\begin{gathered}
D R E C_{-} C_{i, t}=\omega_{0}+\omega_{1} D B O V_{-} N 1_{i, t}+\omega_{2} D B O V_{-} N 2_{i, t}+\omega_{3} D B O V_{-} N M_{i, t} \\
+\omega_{n} \text { VARCONTROL } L_{i, t}+\varepsilon_{i, t}
\end{gathered}
$$


Em que: DREC_C $\mathrm{C}_{\mathrm{i}, \mathrm{t}}=$ variável dummy igual a 1 se as ações da empresa $i$ receberam recomendações de compra no ano $t$; e igual a 0 ; caso contrário. Outras variáveis dummies foram utilizadas com o objetivo de capturar o efeito dos níveis de governança, tais como: DBOV_N1 representa a prática de Governança Corporativa classificada como Nível 1; DBOV_N2 representa a prática de Governança Corporativa classificada como Nível 2; e DBOV_NM representa a prática de Governança Corporativa classificada como Novo Mercado; utilizou-se um conjunto de variáveis de controle representadas por VARCONTROL $\mathrm{i}_{\mathrm{i}, \mathrm{t}}$ Além disso, $\omega$ representa os coeficientes do modelo; $i$ e $t$ representam a empresa e o ano, respectivamente; e $\varepsilon_{i t}=$ valor termo de erro da regressão.

O segundo modelo testado foi o Modelo 2 - Recomendação de Manutenção. O objetivo deste modelo também é testar a hipótese $\mathrm{H}_{1}$. Ou seja, verificar se as empresas que adotam práticas diferenciadas de governança corporativa têm mais probabilidade de receber recomendações de manutenção.

$$
\begin{aligned}
D R E C M_{i, t}= & \beta_{0}+\beta_{1} D B O V_{-} N 1_{i, t}+\beta_{2} D B O V_{-} N 2_{i, t}+\beta_{3} D B O V_{-} N M_{i, t} \\
& +\beta_{n} \text { VARCONTROL } L_{i, t}+\varepsilon_{i, t}
\end{aligned}
$$

Em que: DREC_M $\mathrm{M}_{\mathrm{i}, \mathrm{t}}=$ variável dummy igual a 1 se as ações da empresa $i$ tiveram recomendações de manutenção no exercício social $t$ e igual a 0 , caso contrário. Outras variáveis dummies foram utilizadas com o objetivo de capturar o efeito dos níveis de governança, tais como: DBOV_N1 representa a prática de Governança Corporativa classificada como Nível 1; DBOV_N2 representa a prática de Governança Corporativa classificada como Nível 2; e DBOV_NM representa a prática de Governança Corporativa classificada como Novo Mercado; utilizou-se um conjunto de variáveis de controle representadas por VARCONTROL $\mathrm{L}_{\mathrm{i}, \mathrm{t}}$. Além disso, $\omega$ representa os coeficientes do modelo; $i$ e $t$ representam a empresa e o ano, respectivamente; e $\varepsilon_{\text {it }}=$ valor termo de erro da regressão.

O terceiro modelo desenvolvido nesta pesquisa foi o Modelo 3 - Recomendação de Venda. O objetivo deste modelo é testar a hipótese $\mathrm{H}_{1}$. Ou seja, verificar se as empresas que não adotam práticas diferenciadas de governança corporativa têm mais probabilidade de receber recomendações de venda dos analistas de investimento.

$$
\begin{aligned}
D R E C_{-} V_{i, t}= & \delta_{0}+\omega_{1} D B O V_{-} N 1_{i, t}+\delta_{2} D B O V_{-} N 2_{i, t}+\delta_{3} D B O V_{-} N M_{i, t} \\
& +\delta_{n} \text { VARCONTROL } L_{i, t}+\varepsilon_{i, t}
\end{aligned}
$$

Em que: DREC_ $\mathrm{V}_{\mathrm{i}, \mathrm{t}}=$ Variável dummy igual a 1 se as ações da empresa $i$ tiveram recomendações de venda no exercício social $t$ e igual a 0 caso contrário. Outras variáveis dummies foram utilizadas com o objetivo de capturar o efeito dos níveis de governança, tais como: DBOV_N1 representa a prática de Governança Corporativa classificada como Nível 1; DBOV_N2 representa a prática de Governança Corporativa classificada como Nível 2; e DBOV_NM representa a prática de Governança Corporativa classificada como Novo Mercado; utilizou-se um conjunto de variáveis de controle representadas por VARCONTROL $\mathrm{i}, \mathrm{t}$. Além disso, $\omega$ representa os coeficientes do modelo; $i$ e $t$ representam a empresa e o ano, respectivamente; e $\varepsilon_{\text {it }}=$ valor termo de erro da regressão.

\section{ANÁLISE DOS RESULTADOS}

\subsection{Estatística Descritiva}

A partir dos dados coletados, na Tabela 6 apresenta-se o resumo da estatística descritiva das variáveis utilizadas nos modelos. A descrição das variáveis DREC_C, DREC_M, DREC_V, DBOV_N1, DBOV_N2, e DBOV_NM não consta na Tabela 6, pois são variáveis dummies, com mínimo igual a zero e máximo igual a 1. 
Tabela 6

Estatística descritiva das variáveis

\begin{tabular}{lccccccc}
\hline \multicolumn{1}{c}{ Variável } & Média & $\begin{array}{c}\text { Desvio } \\
\text { padrão }\end{array}$ & Mínimo & P25 & P50 & P75 & Máximo \\
\hline QANALIST & 2,50 & 3,69 & 0,00 & 0,00 & 0,00 & 4,00 & 13,00 \\
TAMCOR & 7,65 & 4,63 & 1,00 & 3,00 & 8,00 & 11,00 & 17,00 \\
TAMEMP & 8,02 & 9,39 & 1,02 & 3,95 & 5,81 & 7,41 & 11,07 \\
RENTABLIDADE & $-0,05$ & 0,50 & $-1,89$ & $-0,02$ & 0,02 & 0,07 & 0,30 \\
LIQUIDEZ & 14,17 & 13,74 & 0,04 & 0,97 & 14,09 & 14,55 & 15,73 \\
\hline
\end{tabular}

A seguir são apresentados os modelos econométricos desenvolvidos neste estudo. O objetivo destes modelos é verificar se as empresas que aderiram a um dos níveis de governança da B3 afetaram a recomendação dos analistas, testando assim, a hipótese de pesquisa.

\subsection{Modelo DREC_C (1)}

Esse modelo tem por objetivo analisar a relação multivariada entre a variável dependente DREC_C (recomendação de compra) e as demais variáveis do modelo. Os resultados apresentados na Tabela 7 demonstram que, pelo $p$-valor da estatística LR Chi2, há pelo menos um coeficiente cujo valor seja diferente de zero a um nível de significância de 1\%, demonstrando a relevância conjunta do modelo. Além disso, pode-se verificar que, ao nível de significância de 5\%, as variáveis TAMEMP e DBOV_NM apresentaram significância. Estes resultados estão em conformidade com os obtidos em estudos anteriores (Dalmácio, 2009; Dalmácio et al., 2010; Dalmácio et al., 2013). Ao nível de $10 \%$ de confiança, as variáveis DBOV_N2 e LIQUIDEZ foram significativas. Por outro lado, as variáveis QANALIST, TAMCOR, DBOV_N1 e RENTABILIDADE não apresentaram significância no modelo proposto.

Tabela 7

Resultados do modelo DREC_C

\begin{tabular}{|c|c|c|c|}
\hline \multicolumn{4}{|c|}{$D R E C_{-} C_{i, t}=\omega_{0}+\omega_{1} D B O V_{-} N 1_{i, t}+\omega_{2} D B O V_{-} N 2_{i, t}+\omega_{3} D B O V_{-} N M_{i, t}+\omega_{n} V A R C O N T R O L_{i, t}+\varepsilon_{i, t}$} \\
\hline DREC_C & Odds Ratio & Std. Err. & $(\mathbf{O R}-1) * 100$ \\
\hline QANALIST & 0,967536 & 0,0227335 & \\
\hline TAMCOR & 1,016795 & 0,0203767 & \\
\hline TAMEMP & $1,456725 * *$ & 0,8126812 & 45,6725 \\
\hline DBOV_N1 & 1,036623 & 0,2666208 & 3,6623 \\
\hline DBOV_N2 & $1,691601 *$ & $\mathbf{0 , 5 0 8 4 8 3 9}$ & 69,1601 \\
\hline DBOV_NM & $1,509224 * *$ & 0,2875964 & 50,9224 \\
\hline RENTABILIDADE & 1,006474 & 0,3313981 & 0,6474 \\
\hline LIQUIDEZ & $1,00987 *$ & 0,57696154 & 0,987 \\
\hline Dummy SETOR & Sim & & \\
\hline N. Obs. & 3.112 & & \\
\hline LR chi2 & 21,02 & & \\
\hline Prob >chi 2 & 0,0000 & & \\
\hline Pseudo R2 & 0,1227 & & \\
\hline
\end{tabular}

$* * * \mathrm{p}<0,01, * * \mathrm{p}<0,05, * \mathrm{p}<0,10$

Para análise dos resultados, calculou-se a probabilidade de ocorrência, a partir do Odds Ratio (razão de chances), conforme a Tabela 7. As maiores empresas (TAMEMP) têm 45,6725\% a mais de chances de serem recomendadas para compra do que as menores. Ademais, em relação às empresas que estão listadas nos níveis de governança corporativa da B3: 1) Empresas listadas no Nível 2 da B3 têm 69,1601\% a mais de chances de terem recomendação de compra; e 2) Empresas listadas no Novo Mercado da B3 têm 50,9224\% a mais de chances de terem recomendação de compra. 


\subsection{Modelo DREC_M (2)}

Esse modelo tem por objetivo analisar a relação multivariada entre a variável dependente DREC_M (recomendação de manutenção) e as demais variáveis do modelo. Os resultados apresentados na Tabela 8 demonstram que, pelo $p$-valor da estatística LR Chi2, há pelo menos um coeficiente cujo valor seja diferente de zero a um nível de significância de $1 \%$, demonstrando a relevância conjunta do modelo. Além disso, pode-se verificar que a única variável que apresentou significância, ao nível de 5\%, para determinar a probabilidade de a empresa ter ou não recomendação de manutenção foi TAMEMP. Algumas pesquisas, ao analisar o efeito dos mecanismos de governança na acurácia dos analistas, também encontraram resultado similar (Dalmácio, 2009; Dalmácio et al., 2010; Dalmácio et al., 2013).

\section{Tabela 8}

Resultados do modelo DREC_M

\begin{tabular}{cccc}
\hline$D R E C_{-} M_{i, t}=\beta_{0}+\beta_{1} D B O V_{-} N 1_{i, t}+\beta_{2} D B O V_{\_} N 2_{i, t}+\beta_{3} D B O V \_N M_{i, t}+\beta_{n} V A R C O N T R O L_{i, t}+\varepsilon_{i, t}$ \\
\hline DREC_M & Odds Ratio & Std. Err. & $(\mathbf{O R - 1})^{* 100}$ \\
\hline QANALIST & 1,024047 & 0,0240497 & 2,4047 \\
TAMCOR & 1,012147 & 0,0203412 & 1,2147 \\
TAMEMP & $\mathbf{0 , 9 9 9 9 8 0 2 *}$ & $\mathbf{0 , 0 7 4 8 0 9 8}$ & $\mathbf{9 , 8 9 9 8 0 2}$ \\
DBOV_N1 & 0,8253566 & 0,2128668 & $-17,46434$ \\
DBOV_N2 & 0,7002857 & 0,2114663 & $-29,97143$ \\
DBOV_NM & 0,7805735 & 0,1485183 & $-21,94265$ \\
RENTABILIDADE & 1,133923 & 0,3829356 & 13,3923 \\
LIQUIDEZ & 0,100999999 & 0,001246351 & $-89,9000001$ \\
Dummy SETOR & Sim & & \\
N. Obs. & 3.112 & \\
LR chi2 & 10,32 & & \\
Prob $>$ chi2 & 0,0000 & & \\
Pseudo R2 & 0,0413 & &
\end{tabular}

Para análise dos resultados, calculou-se a probabilidade de ocorrência, a partir do Odds Ratio (razão de chances), conforme a Tabela 8. De acordo com os resultados da regressão logística, as maiores empresas (TAMEMP) têm 9,899\% a mais de chances de serem recomendadas para manutenção do que as menores. Além disso, verifica-se que para o modelo proposto, ter adotado níveis diferenciados de governança não afetou a probabilidade de a empresa receber recomendação de manutenção.

\subsection{Modelo DREC_V}

Esse modelo tem por objetivo analisar a relação multivariada entre a variável dependente DREC_V (recomendação de venda) e as demais variáveis do modelo. Os resultados apresentados na Tabela 9 demonstram que, pelo p-valor da estatística LR Chi2, há pelo menos um coeficiente cujo valor seja diferente de zero a um nível de significância de 1\%, demonstrando a relevância conjunta do modelo. Também, verifica-se que a única variável com significância, ao nível de 5\%, para determinar a probabilidade de a empresa ter ou não recomendação de venda foi DBOV_N1.

Para a análise dos resultados, calculou-se a probabilidade de ocorrência, a partir do Odds Ratio (razão de chances), conforme a Tabela 9. De acordo com os resultados da regressão logística, empresas listadas no Nível 1 da B3 têm 264,79\% a mais de chances de terem recomendação de venda (considerando um nível de significância de 5\%).

Estes resultados evidenciam que os níveis diferenciados de governança corporativa afetam a recomendação dos analistas. Desta forma, este resultado evidencia que quando as empresas adotam 
níveis diferenciados de governança da B3, elas emitem um sinal positivo para os analistas. Este resultado está alinhado com estudos anteriores (Dalmácio, 2009; Dalmácio et al., 2010; Dalmácio et al., 2013).

Tabela 9

Resultados do modelo DREC_V

\begin{tabular}{cccc}
\hline \multicolumn{2}{c}{ DREC_ $V_{i, t}=\beta_{0}+\beta_{1} D B O V_{-} N 1_{i, t}+\beta_{2} D B O V_{-} N 2_{i, t}+\beta_{3} D B O V_{-} N M_{i, t}+\beta_{n} V A R C O N T R O L_{i, t}+\varepsilon_{i, t}$} \\
\hline DREC_V & Odds Ratio & Std. Err. & $(\mathbf{O R}-\mathbf{1}) * \mathbf{1 0 0}$ \\
\hline QANALIST & 1,054509 & 0,0823563 & 5,4509 \\
TAMCOR & $\mathbf{1 , 8 9 3 5 5 1 4 *}$ & $\mathbf{0 , 0 5 7 5 6 1 7}$ & $\mathbf{8 9 , 3 5 5 1 4}$ \\
TAMEMP & 0,9999463 & 0,00484765 & $-0,00537$ \\
DBOV_N1 & $\mathbf{3 , 6 4 7 9 3 1 * *}$ & $\mathbf{2 , 6 2 5 1 2}$ & $\mathbf{2 6 4 , 7 9 3 1}$ \\
DBOV_N2 & 0,6917119 & 0,7880436 & $-30,82881$ \\
DBOV_NM & 1,073654 & 0,614946 & 7,3654 \\
RENTABILIDADE & 1,665368 & 2,355599 & 66,5368 \\
LIQUIDEZ & 1,0986345 & 0,093757 & 9,86345 \\
Dummy SETOR & Sim & & \\
N. Obs. & 3.112 & \\
LR chi2 & 10,88 & \\
Prob>chi2 & 0,0000 & \\
Pseudo R2 & 0,0583 & \\
\hline
\end{tabular}

$* * * \mathrm{p}<0,01, * * \mathrm{p}<0,05, * \mathrm{p}<0,10$

\section{CONCLUSÃO}

O objetivo geral deste artigo foi analisar, a partir da Teoria da Sinalização, se estar nos níveis diferenciados de governança corporativa afeta as recomendações dos analistas de investimento (compra, manutenção e venda) do mercado brasileiro. Alguns artigos analisaram o efeito dos níveis de governança. Por exemplo, Silva et al. (2011) analisou o efeito da governança no risco das empresas. Outros estudos investigaram o impacto da governança no desempenho das empresas (Oliveira, 2019; Marcos et al., 2018). Por outro lado, Marquiori (2019) analisou se a governança corporativa cria valor para a firma. Assim, o presente estudo contribui para a literatura ao propor que adotar um dos níveis de governança da B3 pode ser um sinal para o mercado.

Desta forma, a partir da Teoria da Sinalização (Spence, 1973), constatou-se que a governança corporativa pode representar um sinal ao mercado, capaz de influenciar as recomendações emitidas por esses analistas. Reforça-se, consequentemente, as evidências encontradas por Dalmácio (2009), Dalmácio et al. (2010) e Dalmácio et al. (2013), de que a governança representa um parâmetro na alteração da probabilidade condicional a qual determina as crenças, tanto dos analistas na formulação de suas previsões e recomendações, quanto dos investidores na escolha de seus investimentos. Como as pesquisas anteriores (Cowles, 1933; Jaffe \& Mahoney, 1999; Desai et al., 2000) não são conclusivas a respeito da efetividade das recomendações dos analistas, procurou-se verificar a influência da governança nessas recomendações (de compra, manutenção e venda).

Como proxy para a adoção de práticas diferenciadas de governança corporativa, no Brasil, foram utilizados os níveis diferenciados de governança corporativa da B3 (Nível 1, Nível 2 e Novo Mercado). As recomendações de compra, manutenção e venda foram coletadas da base de dados da Thomson Reuters One - Advanced Analytics ${ }^{\circledR}$, emitidas no período de 2010 a 2016. O relacionamento entre as recomendações dos analistas e a adoção de práticas diferenciadas de governança corporativa foi testado, alternando-se as variáveis dependentes (recomendação de compra, recomendação de manutenção e recomendação de venda), em função das variáveis independentes e de controle. 
Para as análises foi utilizada a regressão logística, associada à Razão de Chances (OddsRatio), a fim de testar quais são as chances das empresas que adotam práticas diferenciadas de governança corporativa receberem mais recomendações de compra e manutenção dos analistas de investimento. Também foram analisadas as chances das empresas que não adotam práticas diferenciadas de governança corporativa receberem mais recomendações de venda desses analistas.

Os resultados obtidos, por meio da utilização do modelo 1 (recomendação de compra), mostram que empresas que adotam o DBOV_N2 de governança corporativa têm $69,16 \%$ a mais de chances de terem recomendação de compra. Empresas listadas no Novo Mercado da B3 têm 50,92\% a mais de chances de terem recomendação de compra. Assim, adotar níveis diferenciados de governança corporativa afeta a probabilidade de a empresa receber recomendação de compra por parte dos analistas.

No caso da utilização do modelo 2 (recomendação de manutenção), os resultados sugerem que aderir níveis diferenciados de governança corporativa não afeta a probabilidade de o analista recomendar a manutenção. Por outro lado, no que se refere à recomendação de venda, os resultados mostram que empresas listadas no DBOV_N1 da B3 têm maior chance de receber recomendação de venda por parte dos analistas. Portanto, pode-se inferir que aderir a níveis diferenciados de governança corporativa afeta a recomendação de compra e de venda. Ou seja, somente para a recomendação de compra e venda a informação de governança corporativa é relevante para a decisão do analista.

Pelos resultados obtidos, por meio da utilização do modelo 1 (recomendação de compra), há evidências de que existe uma conciliação entre a Teoria da Sinalização e a governança corporativa, sustentando a afirmação de que a adoção de práticas diferenciadas de governança corporativa pode, no mercado brasileiro, representar um sinal positivo capaz de influenciar a atividade dos analistas. Tais resultados reforçam os achados de Dalmácio (2009), Dalmácio et al. (2010) e Dalmácio et al. (2013). Dessa forma, recomenda-se que a adoção de práticas diferenciadas de governança corporativa seja valorizada por analistas e investidores do mercado brasileiro.

Ou seja, se os analistas e investidores, ainda, não usam informações sobre a governança corporativa das empresas, em seus processos de tomada de decisão sobre investimentos, poderiam refletir sobre a inclusão dessa variável em seus modelos decisórios, visto que a mesma, além de conferir maior eficácia às previsões, de certa forma, também influencia as recomendações, principalmente, de compra e venda de ações das empresas brasileiras.

Além disso, no modelo 1, pode-se observar que no nível de governança da B3 em que há maior exigência (Novo Mercado), maiores são as chances de as empresas serem recomendadas para compra. De um modo geral, as evidências encontradas sugerem que a adoção de boas práticas de governança traz benefícios para o mercado, em especial, para os investidores. Os resultados também reforçam a importância das normas, regulamentos e/ou recomendações, sobre a necessidade de adoção de práticas diferenciadas de governança, emitidas por instituições, tais como: B3, Comissão de Valores Mobiliários (CVM), Banco Mundial, Instituto Brasileiro de Governança Corporativa (IBGC), Secretaria de Previdência Complementar (SPC), Superintendência de Seguros Privados (SUSEP), Banco Nacional de Desenvolvimento Econômico e Social (BNDES), Fundo Monetário Internacional (FMI), em virtude dos benefícios proporcionados ao mercado brasileiro.

Algumas limitações de pesquisa precisam ser consideradas. A principal é o número limitado de empresas brasileiras de capital aberto que são acompanhadas por analistas de mercado e disponíveis no sistema da Thomson Reuters One - Advanced Analytics ${ }^{\circledR}$. Assim, a amostra selecionada foi intencional e não probabilística. Ou seja, foram analisadas empresas que eram acompanhadas por analistas e estavam disponíveis na base de dados do sistema Thomson Reuters One - Advanced Analytics ${ }^{\circledR}$. Portando, é preciso ter cautela ao tentar generalizar os resultados obtidos, pois as evidências se referem à amostra utilizada.

Ademais, vale destacar que os resultados obtidos são baseados nos níveis de governança da B3, que serviram de proxies para a adoção de práticas diferenciadas de governança pelas empresas 
brasileiras. O estudo está centrado na investigação da influência da adoção de práticas diferenciadas de governança corporativa sobre as recomendações dos analistas, considerando as variáveis utilizadas nos modelos, portanto, a influência de outros fatores, além dos explicitados, foi desconsiderada.

Portanto, ao apontar algumas implicações positivas da adoção de práticas diferenciadas de governança corporativa por parte das empresas brasileiras de capital aberto, espera-se que as evidências encontradas, por meio desta pesquisa, possam trazer contribuições para a literatura nacional e internacional sobre a relação entre governança e recomendações dos analistas, mas, também, para o mercado de capitais brasileiro (analistas, investidores, agências de rating, órgãos reguladores, para as próprias empresas, dentre outros).

\section{AGRADECIMENTO}

Os autores agradecem ao Conselho Nacional de Desenvolvimento Científico e Tecnológico (CNPq) pelo financiamento da pesquisa.

\section{REFERÊNCIAS}

Ahmad-Zaluki, N. A., \& Wan-Hussin, W. N. (2010). Corporate governance and earnings forecasts accuracy. Asian Review of Accounting, 18 (1), 50-67. DOI: https://doi.org/10.1108/JFRA-12-2019-0165

Akerlof, G. (1970). The market for 'lemons': Qualitative uncertainly and the market mechanism. Quarterly Journal of Economics, 84(3), 488-500. DOI: https://doi.org/10.1016/B978-0-12-214850-7.50022-X

Bhat, G., Hope, O. K., \& Kang, T. (2006). Does corporate governance transparency affect the accuracy of analyst forecasts? Accounting and Finance, 46, 715-732. DOI: https://doi.org/10.1111/j.1467629X.2006.00191.x

Barber, B., Lehavy, R., \& Trueman, B. (2000). Are All brokerage houses created equal? Testing for systematic differences in the performance of brokerage houses stock recommendations. Working Paper. http://citeseerx.ist.psu.edu/viewdoc/summary?doi=10.1.1.15.9732.

Barber, B., Lehavy, R., \& Trueman, B. (2001). Can investors profit from the prophets? Security analysts recommendations and stock returns. The Journal of Finance, 56(2), 531-563. DOI: https://doi.org/10.1111/0022-1082.00336

Barber, B., Lehavy, R., McNichols, M. \& Trueman, B. (2006). Buys, holds and sell: the distribution of investment banks' stock ratings and the implications for the profitability of analysts' recommendations. Journal of Accounting and Economics, 41(1-2), 87-117. DOI: https://doi.org/10.1016/j.jacceco.2005.10.001

Boff, L. H., Procianoy, J. L., \& Hoppen, N. (2006). O Uso de informações por analistas de investimento na avaliação de empresas: À procura de padrões. RAC. Revista de Administração Contemporânea, Rio de Janeiro, 10(4), 169-192. DOI : https://doi.org/10.1590/S1415-65552006000400009

Brasil, Bolsa, Balcão (B3). Níveis diferenciados de governança corporativa. 2019. http://www.b3.com.br/pt_br/>. Acesso em: 10 jul. 2019.

Byard, D., Li, Y., \& Weintrop, J. (2006). Corporate governance and the quality of financial analysts' information? Journal of Accounting and Public Policy, 25, 609-625. DOI: https://doi.org/10.1016/j.jaccpubpol.2006.07.003

Chiang, H. T., \& Chia, F. (2005). Analyst's financial forecast accuracy and corporate transparency. Allied Academies International Conference, 10 (1), 9-14. DOI: https://doi.org/10.1111/j.1467629X.2006.00191.X

Chung, K. H., \& Jo, H. (1996). The impact of security analysts' monitoring and marketing functions on the market value of firms. Journal of Financial and Quantitative Analysis, 31(4), 493-512. DOI: https://doi.org/10.2307/2331357

Cliff, T., \& Denis, D. J. (2004). Do initial public offering firms purchase analyst coverage with underpricing? The Journal of Finance, 59(6), 2871-2901. DOI: https://doi.org/10.1111/j.15406261.2004.00719.x

Cowles. A. (1933). Can stock market forecasters forecast? Econometrica, 1(3), 309-324. DOI: https://doi.org/0012-9682(193307)1:3<309:CSMFF>2.0.CO;2-S 
Dalmácio, F. Z. (2009). Mecanismos de governança e acurácia das previsões dos analistas do mercado brasileiro: Uma análise sob a perspectiva da teoria da sinalização. Tese de Doutorado em Controladoria e Contabilidade, Universidade de São Paulo, São Paulo, SP, Brasil. DOI: https://doi.org/10.11606/T.12.2009.tde-17122009-171118

Dalmácio, F. Z., Lopes, A. B., \& Rezende, A. J. (2010). Governança corporativa e acurácia das previsões individuais dos analistas: uma análise no mercado brasileiro. Anais do Congresso USP Controladoria e Contabilidade, São Paulo, SP, Brasil, 10.

Dalmácio, F. Z., Lopes, A. B., Rezende, A. J., \& Sarlo Neto, A. (2013). Uma análise da relação entre governança corporativa e acurácia das previsões dos analistas do mercado brasileiro. RAM. Revista de Administração Mackenzie (Online), 14(5), 104-139.

Desai, H. H., Liang, B., \& Singh, A. K. (2000). Do all-stars shine? Evaluation of analyst recommendations. Financial Analyst Journal, 56(3), 20-29. DOI: https://doi.org/10.2469/faj.v56.n3.2357

Desai, H. H., \& Jain, P. C. (1995). An analysis of the recommendations of the "superstar" money managers at Barron's annual roundtable. The Journal of Finance, 50(4), 1257-1273. DOI: https://doi.org/10.1111/j.1540-6261.1995.tb04057.x

Farooq, O., \& Amrani, H. (2013). Corporate governance and dispersion in analysts' recommendations: Pre and post-crisis analysis from Asian emerging markets. International Journal Business Governance and Ethics, 8(1), 1-17. DOI: https://doi.org/10.1504/IJBGE.2013.052739

Franco, D. (2002). Projeções de lucros sistematicamente exageradas: Um estudo para o Brasil. RBE. Revista Brasileira de Economia, Rio de Janeiro, 56(4), 591-603. DOI: https://doi.org/10.1590/S003471402002000400003

Groysberg, B., Healy, P., Serafeim, G., \& Shanthikumar, D. (2013). The stock selection and performance of buy-side analysts. Management Science, 59(5), 1062-1075. DOI: https://doi.org/10.1287/mnsc.1120.1619

Hair, J., Anderson, R., Tatham, R., \& Black, W. (1998). Multivariate data analysis. 5. ed. New Jersey: Prentice Hall.

Healy, P. M., \& Palepu, K. G. (2001). Information asymmetry, corporate disclosure, and the capital markets: A review of the empirical disclosure literature. Journal of Accounting and Economics, 31(1-3), 405440. DOI: https://doi.org/10.1016/S0165-4101(01)00018-0

Jaffe, J. F., \& Mahoney, J. M. (1999). The performance of investment newsletters. Journal of Financial Economics, 53(2), 289-307. DOI: https://doi.org/10.1016/S0304-405X(99)00023-9

Jegadeesh, N., \& Kim, W. (2006). Value of analyst recommendations: International evidence. Journal of Financial Markets, 9(3), 274-309. DOI: https://doi.org/10.1016/j.finmar.2006.05.001

Jensen, M. C., \& Meckling, W. H. (1976). Theory of the firm: Managerial behavior, agency costs and ownership structure. Journal of Financial Economics, 3(4), 305-360. DOI: https://doi.org/10.1016/0304-405X(76)90026-X

Larcker, D., Richardson, S. A., \& Tuna, I. A. (2007). Corporate governance, accounting outcomes, and organizational performance. The Accounting Review, 82(4), 963-1008. DOI: https://doi.org/10.2308/accr.2007.82.4.963

Lin, J., \& Tai, V. W. (2013). Corporate governance and analyst behavior: Evidence from an emerging market. Asia-Pacific Journal of Financial Studies, 42(2), 228-261. DOI: https://doi.org/10.1111/ajfs.12013

Lopes, A. B. (2008). The relation between firm -specific corporate governance, cross-listing and the informativeness of accounting numbers in Brazil. Thesis [Doctor of Philosophy Degree in The Faculty of Humanities]. Manchester Business School - University of Manchester.

Marcos, C., Nascimento, J. C., Nez, E., \& Kroenke, A. (2018). Desempenho econômico-financeiro e o grau de internacionalização das empresas listadas no Novo Mercado da B3. Revista de Contabilidade e Controladoria, 2(10), 91-105. DOI: http://dx.doi.org/10.5380/rc\&c.v10i2.62879

Marquiori, L. (2019). Governança gera valor? Teste de evento de migração de nível de governança corporativa na B3. Dissertação de mestrado, Fundação Getúlio Vargas, São Paulo, SP, Brasil.

Martinez, A. L. (2004a). Analisando os analistas: Estudo empírico das projeções de lucros e das recomendações dos analistas de mercado de capitais para as empresas brasileiras de capital aberto. Tese de Doutorado em Administração de Empresas, Escola de Administração de Empresas de São Paulo, Fundação Getúlio Vargas, São Paulo, SP, Brasil. 
Martinez, A. L. (2004b). Análise da surpresa dos analistas ao anúncio dos resultados contábeis: evidências empíricas para as companhias abertas brasileiras. Anais do Congresso USP de Controladoria e Contabilidade, São Paulo, SP, Brasil, 4.

Martinez, A. L. (2007). Ganhos e perdas com os profetas do mercado: O valor das recomendações dos analistas. Anais do Congresso da Associação Nacional dos Programas de Pós-Graduação em Ciências Contábeis, Gramado, RS, Brasil, 1.

Martins, G. A., \& Theóphilo, C. R. (2007). Metodologia da investigação científica para ciências sociais aplicadas. São Paulo: Atlas.

Morris, R. D. (1987). Signalling, agency theory and accounting policy choice. Accounting and Business Research, 18(69), 47-69. DOI: https://doi.org/10.1080/00014788.1987.9729347

Moyer, R. C., Chatfield, R. E., \& Sisneros, P. H. (1989). Security analyst monitoring activity: Agency costs and information demands. Journal of Financial and Quantitative Analysis, 24(4), 503-512. DOI: https://doi.org/10.2307/2330982

Oliveira, D. D. M. (2019). Desempenho e governança corporativa: Uma análise econômico-financeira das empresas que aderiram ao segmento "Bovespa Mais" da B3 S.A. Pensar Contábil, 74(21), 24-33.

Shleifer, A., \& Vishny, R. (1997). A survey on corporate governance. The Journal of Finance, 52(2), $737-$ 783. DOI: https://doi.org/10.1111/j.1540-6261.1997.tb04820.x

Silva, W. V., Silveira, S. A. A., Corso, J. M. D., \& Stadler, H. (2011). A influência da adesão às práticas de governança corporativa no risco das ações de empresas de capital aberto. Revista Universo Contábil, 4(7), 82-97. DOI: https://doi.org/ 10.4270/RUC.2011432

Silveira, A. D. M. (2004). Governança corporativa e a estrutura de propriedade: Determinantes e relação com o desempenho das empresas no Brasil. Tese de Doutorado em Administração, Universidade de São Paulo, São Paulo, SP, Brasil.

Spence, A. Michael. (1973). Job market signaling. The Quarterly Journal of Economics, 83(3), 355-374.

Womack, K. (1996). Do brokerage analysts' recommendation have investment value? Journal of Finance, 51(1), 137-167.

Wooldridge, J. M. (2010). Econometric analysis of cross section and panel data. 2. ed. Cambridge: Mit Press.

Yin, Y., \& Tan, B. (2017). Analyst's ability, media selection and investor interests: Evidence from China. China Finance Review International, 7(1), 67-84. DOI: https://doi.org/10.1108/CFRI-06-2016-0049

$\mathrm{Yu}, \mathrm{F}$. (2005). Essays on governance and earnings management. Dissertation for the degree of Doctor of Philosophy, University of Chicago, Chicago, Illinois.

$\mathrm{Yu}, \mathrm{M}$. (2011). Analyst recommendations and corporate governance in emerging markets. International Journal of Accounting and Information Management, 19(1), 34-52. DOI:

https://doi.org/10.1108/18347641111105926 